\title{
Impact of metabolic syndrome on the progression of coronary calcium and of coronary artery disease assessed by repeated cardiac computed tomography scans
}

Lee Kyung Kim ${ }^{1,2 \dagger}$, Ji Won Yoon ${ }^{1,3 \dagger}$, Dong-Hwa Lee ${ }^{1,2}$, Kyoung Min Kim², ${ }^{1,2}$ Sung Hee Choi ${ }^{1,2}$, Kyong Soo Park' , Hak Chul Jang ${ }^{1,2}$, Min-Kyung Kim ${ }^{1,3}$, Hyo Eun Park ${ }^{1,3}$, Su-Yeon Choi ${ }^{1,3^{*}}$ and Soo Lim ${ }^{1,2^{*}}$

\begin{abstract}
Background: It is not clear how severe metabolic syndrome (MS) affects the development of coronary atherosclerosis.

Methods: This was an observational, retrospective cohort study with Koreans who received health check-ups voluntarily. A total of 2426 subjects had baseline and follow-up coronary artery calcium score (CACS) data. Among them, 1079 had coronary computed tomography angiography (CCTA) data. We compared baseline CACS and any progression in subjects with and without MS. A more detailed analysis was conducted for coronary artery disease (CAD), which was defined by coronary artery stenosis ( $\geq 50 \%$ ), multivessel involvement, and coronary plaques in those patients with CCTA data.

Results: At baseline, subjects with MS (34.0\%, $n=825)$ had higher CACS and more significant coronary artery stenosis, multivessel involvement, and atheromatous plaques than those without MS ( $P<0.05$ for all). In the follow-up (median 1197 days), subjects with MS showed significant increases in CACS and progression of CAD compared with counterparts without MS, in parallel with the numbers of MS components. Finally, MS was a significant predictor for the progression of CACS (hazard ratio 1.32; $95 \%$ confidence interval 1.06-1.64) and progression of coronary artery stenosis and/or development of vulnerable plaque (hazard ratio 1.47, 95 \% confidence interval 1.01-2.15) after adjusting for other cardiovascular risk factors.
\end{abstract}

Conclusions: Subjects with MS showed progression of CAD as assessed by CACS and CCTA over $~ 3$ years. Therefore, more vigilant screening for coronary vascular health is needed among those with MS.

Keywords: Metabolic syndrome, Coronary artery calcium, Coronary artery disease, Longitudinal cohort study

\footnotetext{
*Correspondence: sychoi9@gmail.com; limsoo@snu.ac.kr

†Lee Kyung Kim and Ji Won Yoon contributed equally to this work

${ }^{2}$ Internal Medicine, Seoul National University Bundang Hospital, 300

Gumi-dong, Bundang-Gu, Seongnam 463-707, South Korea

${ }^{3}$ Internal Medicine, Seoul National University Hospital Healthcare System

Gangnam Center, 39th FL. Gangnam Finance Center, 737 Yeoksam-dong,

Gangnam-gu, Seoul 135-984, South Korea

Full list of author information is available at the end of the article
} 


\section{Background}

Cardiovascular disease is the leading cause of deaths worldwide [1]. According to a report of the World Health Organization in 2009, $30 \%$ of all global deaths were attributed to cardiovascular diseases in 2008. It is also estimated that by 2030 , over 23 million people will die from cardiovascular diseases each year $[2,3]$. Thus, there is a major social cost in the management of cardiovascular disease.

Under these circumstances, it is worthwhile to prescreen subjects for subclinical coronary atherosclerosis and manage risk factors to prevent its development and progression. There are several methods of screening for cardiovascular disease, such as electrocardiograms, the ankle-to-brachial blood pressure index, pulse wave velocity, carotid intima-media thickness, $N$-terminal pro-brain natriuretic peptide, the presence of carotid plaques, coronary artery calcium score (CACS), and coronary artery stenosis [4-8].

Among these, CACS is used widely as a screening method [9]. CACS reflects the presence and the extent of coronary atherosclerosis and has an independent association with cardiovascular events [10]. It has been used for individualized risk stratification and for predicting outcomes $[11,12]$. The American Heart Association has suggested that CACS is a reasonable tool to assess the risk of cardiovascular disease [13]. Moreover, repeated CACS measures were found to be useful in predicting cardiovascular outcomes and in assessing the effectiveness of treatments [14-16].

In the recent decade, there has been much progress in technologies in coronary computed tomography angiography (CCTA). This is able to provide more detailed information than CACS, including the degree of stenosis in individual coronary arteries, plaque characteristics, and the extent of any vascular remodeling [17-19]. We have reported that the evaluation of possible coronary artery disease (CAD) with multidetector CCTA is of importance in the early diagnosis of atherosclerosis in asymptomatic patients, particularly for assessing plaque characteristics [20].

Metabolic syndrome (MS) is a disorder, characterized by clusters of cardiovascular risk factors such as visceral obesity, high blood pressure, dyslipidemia, and impaired glucose tolerance [21]. Studies have confirmed that people with MS have an increased risk of cardiovascular morbidity and mortality [22].

There have been several studies supporting the relationship between MS and high burden of CACS [23, 24]. We have reported the association between MS and cardiovascular disease using multidetector CCTA [20]. However, longitudinal follow-up studies investigating the impact of MS on coronary vascular health using CACS or multidetector CCTA are limited [25]. Therefore, the aim of this study was to investigate the association of any progression of CACS and CAD with the severity of MS in a large Korean population, using CACS and CCTA.

\section{Methods \\ Study population}

This study was an observational, retrospective cohort study. Among people ( $n>10,000$ per year) who participated in a routine health check-up at Seoul National University Hospital Healthcare System Gangnam Center, a total of 17,390 subjects who had at least one or more cardiovascular risk factors, or who had atypical chest pain, underwent a CCTA from October 1, 2003 to December 31, 2012. Among them, 14,765 subjects who did not have follow-up CCTA scans until December 2013 were excluded. Next, 18 subjects who had medical histories of coronary revascularization at baseline were excluded. We also excluded those subjects whose CACS or coronary vascular health were not adequately evaluated $(n=97)$ or who had missing data for defining MS $(n=98)$.

Finally, a total of 2426 subjects who had a baseline and a follow-up CACS at least 1 year apart were included in the analysis. The median follow-up period was 1197 days (interquartile range 766.0-1687.5). Among these subjects, 1079 underwent CCTA tests at baseline and a follow-up CCTA test at least 1 year later. In this subgroup, we evaluated the progression of CAD by assessing the degree of coronary artery stenosis and presence of plaques and its characteristics. Selection of the study participants is outlined in Fig. 1.

The study protocol conformed to the ethical guidelines of the Declaration of Helsinki (2013). It was approved by the institutional review board of Seoul National University Hospital (IRB No. H-1501-003-635). All patients underwent multidetector CT after they had agreed to participate in the study and had provided informed consent after being informed of the possible risks of CT scanning.

\section{Anthropometric measurement and laboratory evaluations}

On the day of the CT scan, anthropometric parameters such as height, body weight, waist circumference, and systolic and diastolic blood pressures were measured by trained nurses. Body mass index (BMI) was calculated as weight divided by height in $\mathrm{kg} / \mathrm{m}^{2}$. Waist circumference was measured at the midpoint between the lower costal margin and the iliac crest.

Each subject completed a self-administered questionnaire, which included questions on demographic factors; a medical history covering diabetes mellitus (DM), dyslipidemia, hypertension, coronary revascularization, and drug use; family history of cardiovascular disease; and a 


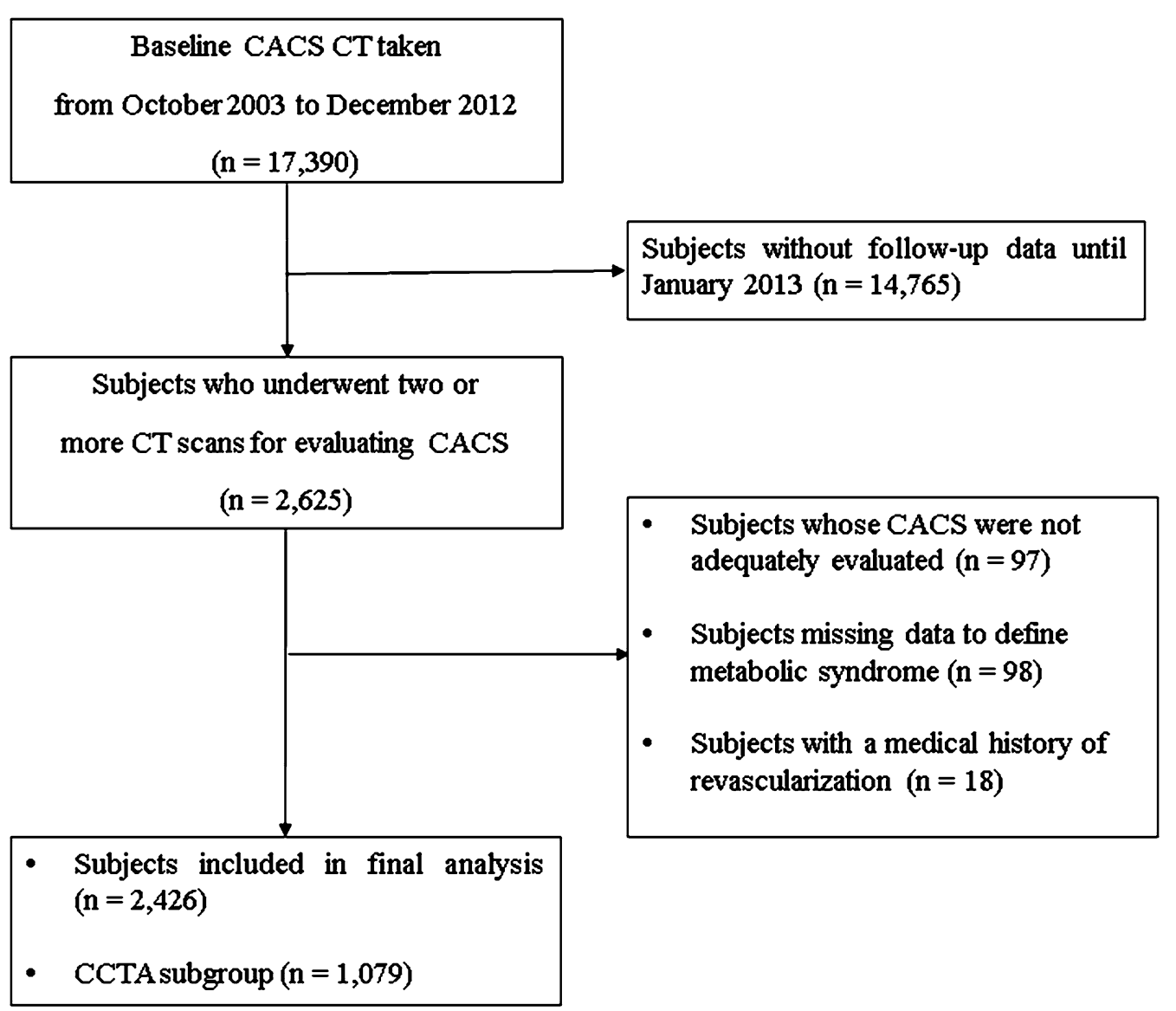

Fig. 1 Selection of study participants

social history including smoking habit, alcohol consumption, and levels of physical activity.

Smoking status was defined as follows: Patients were classified as current smokers if they currently smoked for at least 1 year. Patients were classified as nonsmokers if they had never smoked. Patients were classified as exsmokers if they had smoked but quit. Patients were classified as nondrinkers or current drinkers by determining their average daily alcohol consumption. Nondrinkers were those who had not consumed alcohol within the past 30 days. Physical activity was classified into two categories: none or regular exercise. Regular exercise was defined as exercising more than three times a week (each session should be at least $30 \mathrm{~min}$ ).

For biochemical tests, fasting plasma concentrations of glucose, total cholesterol, triglycerides, high-density lipoprotein (HDL)-cholesterol, and serum high-sensitivity C-reactive protein (hsCRP) were measured using an Architect Ci8200 analyzer (Abbott Laboratories, Abbott Park, IL, USA). Glycated hemoglobin (HbA1c) levels were measured using a COBAS INTEGRA 400 instrument (Roche Diagnostics GmbH, Mannheim, Germany).
Plasma insulin concentrations were measured by radioimmunoassay (Linco, St. Louis, MO, USA). Aspartate/alanine aminotransferase (AST/ALT), gamma-glutamyl transpeptidase $(\gamma \mathrm{GT})$, and creatinine were measured using an Architect Ci8200 analyzer (Abbott Laboratories, Abbott Park, IL, USA). Low-density lipoprotein (LDL)-cholesterol concentration was calculated using the Friedewald equation [26]. In subjects with a triglyceride level $\geq 400 \mathrm{mg} / \mathrm{dL}$, the measured LDL-cholesterol was used for analysis.

The homeostasis model assessment of insulin resistance (HOMA-IR) and pancreatic $\beta$-cell function (HOMA- $\beta$ ) were calculated as described by Matthews et al. [27]. HOMA-IR $=$ fasting insulin $(\mu \mathrm{IU} / \mathrm{mL}) \times$ fasting plasma glucose $(\mathrm{mg} / \mathrm{dL}) / 405$; HOMA- $\beta=360 \times$ fasting insulin $(\mu \mathrm{IU} / \mathrm{mL}) /[$ fasting plasma glucose $(\mathrm{mg} / \mathrm{dL})-63]$.

Diabetes mellitus was defined as a fasting plasma glucose level of $126 \mathrm{mg} / \mathrm{dL}$ (7 mmol/L) or higher or current antidiabetic treatment. Hypertension was defined as systolic/diastolic blood pressure measures greater than 140/90 mmHg or current treatment with antihypertensive medication. Dyslipidemia was defined as an LDL-cholesterol level of $160 \mathrm{mg} / \mathrm{dL}$ or higher or current use of a lipid-lowering agent. 


\section{Evaluation of initial coronary $\mathrm{CT}$ angiography}

In all subjects, coronary $\mathrm{CT}$ was performed either with a 16-slice scanner (Somatom Sensation 16; Siemens Medical Solutions, Forchheim, Germany) or with a 256-slice multidetector CT scanner (Brilliance iCT 256; Philips Medical Systems, Cleveland, OH, USA). A standard scanning protocol was applied, with $128 \times 0.625 \mathrm{~mm}$ section collimation, $0.27 \mathrm{~ms}$ rotation time, $120 \mathrm{kV}$ tube voltage, and $800 \mathrm{~mA}$ tube current. All scans were performed with electrocardiogram-gated dose modulation. Two experienced radiologists-blinded to all clinical information and patient demographics-analyzed all CT scans. The CACS was calculated quantitatively as described by Agatston et al. [28] using dedicated software (Rapidia 2.8; INFINITT, Seoul, South Korea).

Coronary artery stenosis and plaque characteristics were evaluated in the subjects who underwent multidetector CCTA. The coronary lumen cross-sectional diameter was traced at the maximal stenotic site and compared with the mean value for the proximal and distal reference sites. Coronary artery stenosis was evaluated according to five categories as follows: (1) normal or minimal (absence of plaque and no stenosis to plaque with <25\% stenosis); (2) mild ( $25-49 \%$ stenosis); (3) moderate (50-69 \% stenosis); (4) severe stenosis (70$99 \%$ stenosis); and (5) occluded (100\% stenosis) [29, 30]. We recorded the degree of stenosis in each segment for one vessel and finally defined the maximal stenosis as being the grade of stenosis used in analysis for that vessel. Significant stenosis was defined as the presence of at least moderate stenosis. We also defined multivessel involvement when there was the presence of $\geq 50 \%$ stenosis in at least two vessels.

Plaque characteristics were evaluated by arterial segment: left main artery; proximal, middle, and distal segment of left anterior descending artery; left circumferential artery; and right coronary artery. Each plaque was classified as calcified, mixed, or noncalcified type: (1) plaques that contained calcified tissue comprising $\geq 50 \%$ of the plaque area were classified as calcified; (2) plaques with $<50 \%$ calcium in the plaque area were classified as mixed; and (3) plaques without any calcium were classified as noncalcified lesions [31]. If there was any plaque on a segment, we counted it as an atherosclerotic coronary segment. If there was a mixed or a noncalcified plaque, it was defined as a vulnerable atheromatous plaque $[32,33]$ (Additional file 1: Figure S1). Definition of metabolic syndrome

In accordance with modified National Cholesterol Education Program-Adult Treatment Panel III criteria [34, 35], an individual was classified as having MS if he or she had three or more of the flowing five criteria: (1) waist circumference $\geq 90 \mathrm{~cm}$ in men and $\geq 80 \mathrm{~cm}$ in women, using the International Obesity Task Force criteria for the Asian-Pacific population to determine waist circumference criteria [36]; (2) triglyceride levels $\geq 150 \mathrm{mg}$ / dL $(1.7 \mathrm{mmol} / \mathrm{L})$; (3) HDL-cholesterol level $<40 \mathrm{mg} / \mathrm{dL}$ $(1.0 \mathrm{mmol} / \mathrm{L})$ in men and $<50 \mathrm{mg} / \mathrm{dL}(1.3 \mathrm{mmol} / \mathrm{L})$ in women; (4) blood pressure $\geq 130 / 85 \mathrm{mmHg}$ or the use of antihypertensive medication; and (5) fasting glucose level $\geq 100 \mathrm{mg} / \mathrm{dL}(6.1 \mathrm{mmol} / \mathrm{L})$ or the self-reported use of antidiabetic medication (insulin or oral agents).

\section{Assessment of progression of coronary artery disease on cardiac CT}

CACS progression was the main outcome measure of this study, which was assessed according to Berry et al. [37]. In patients with absence of any calcification at baseline $(C A C S=0)$, progression was defined as CACS $>0$ at follow-up. In subjects with a CACS $>0$ and $<100$, progression was defined as an annualized increase of at least 10 Agatston units at follow-up, and for those with a CACS $\geq 100$, an annualized percentage increase of $\geq 10 \%$ at the follow-up was defined as progression.

For secondary outcome 1, progression of coronary artery stenosis was defined by the following conditions: (1) progression in the grade of stenosis at the vessel where the original stenosis was identified; and (2) generation of new stenosis at other segments. For secondary outcome 2 , the development of new vulnerable plaque was defined as when any mixed or noncalcified plaque had developed in one or more segments.

\section{Statistical analysis}

We compared data using Student's $t$ tests for continuous variables and $\chi^{2}$ tests for categorical variables. All data are expressed as the mean \pm standard deviation (SD), median (interquartile range), or number (frequency). Log transformation was used for the statistical analysis of CACS, HOMA-IR, and hsCRP. $x^{2}$ tests with linear-by-linear associations were applied to examine the significance of any linear trend of CAD progression according to the number of MS components. A Cox proportional hazard model was used to investigate any independent effect of MS on the progression of CAD. The analyses were performed using IBM SPSS Statistics for Windows (version 20.0; IBM Corp., Armonk, NY, USA). Data with $P<0.05$ were considered significant.

\section{Results}

\section{Baseline clinical characteristics}

Baseline characteristics and biochemical parameters were compared according to the presence or absence of MS (Table 1). Baseline characteristics according to the gender were presented in the Additional file 1: Table S1. At baseline, 825 subjects had MS (34.0\% of total population). 
Table 1 Baseline clinical and biochemical characteristics in subjects with and without metabolic syndrome (MS)

\begin{tabular}{|c|c|c|c|}
\hline & MS $(n=825)$ & Non-MS $(n=1601)$ & $P$ \\
\hline \multicolumn{4}{|c|}{ Clinical and anthropometric parameters } \\
\hline Age, years & $56.6(7.5)$ & $56.2 \pm 7.2$ & 0.180 \\
\hline No. (\%) of male & $661(80.0)$ & $1278(79.8)$ & 0.873 \\
\hline Height, cm & $167.5(7.6)$ & $166.7(7.4)$ & 0.010 \\
\hline Weight, kg & $73.8(10.3)$ & $66.5(9.1)$ & $<0.001$ \\
\hline $\mathrm{BMI}, \mathrm{kg} / \mathrm{m}^{2}$ & $26.2(2.6)$ & $23.9(2.3)$ & $<0.001$ \\
\hline Waist circumference, cm & $92.7(6.6)$ & $85.9(6.4)$ & $<0.001$ \\
\hline $\mathrm{SBP}, \mathrm{mmHg}$ & $127.5(13.8)$ & $118.5(14.3)$ & $<0.001$ \\
\hline $\mathrm{DBP}, \mathrm{mmHg}$ & $83.8(10.5)$ & $77.8(10.5)$ & $<0.001$ \\
\hline \multicolumn{4}{|l|}{ Biochemical parameters } \\
\hline Fasting glucose, $\mathrm{mmol} / \mathrm{L}$ & $6.37(1.30)$ & $5.49(0.93)$ & $<0.001$ \\
\hline $\mathrm{HbA} 1 \mathrm{c}, \%$ & $6.1(0.8)$ & $5.8(0.6)$ & $<0.001$ \\
\hline Insulin, $\mu \mathrm{U} / \mathrm{mL}$ & $12.3(5.6)$ & $8.5(4.1)$ & $<0.001$ \\
\hline HOMA-IR & $3.48(1.74)$ & $2.12(1.10)$ & $<0.001$ \\
\hline $\mathrm{HOMA}-\beta^{\mathrm{a}}$ & $97.6(57.8)$ & $95.0(54.2)$ & 0.434 \\
\hline AST, IU/L & $28.9(14.0)$ & $24.8(9.3)$ & $<0.001$ \\
\hline$A L T, I U / L$ & $34.3(21.5)$ & $26.2(14.7)$ & $<0.001$ \\
\hline$\gamma G T, I U / L$ & $55.6(59.5)$ & $35.7(30.8)$ & $<0.001$ \\
\hline Creatinine, $\mu \mathrm{mol} / \mathrm{L}$ & $93.70(17.68)$ & $93.70(15.03)$ & 0.884 \\
\hline Total cholesterol, mmol/L & $5.13(0.93)$ & $5.18(0.88)$ & 0.147 \\
\hline Triglyceride, mmol/L & $2.04(104.1)$ & $1.18(0.58)$ & $<0.001$ \\
\hline HDL-cholesterol, mmol/L & $1.21(0.29)$ & $1.42(0.32)$ & $<0.001$ \\
\hline LDL-cholesterol, mmol/L & $3.02(0.87)$ & $3.23(0.81)$ & $<0.001$ \\
\hline $\mathrm{hsCRP}{ }^{\mathrm{a}}, \mathrm{mg} / \mathrm{dL}$ & $0.17(0.42)$ & $0.14(0.44)$ & 0.118 \\
\hline \multicolumn{4}{|l|}{ Comorbidity and lifestyles } \\
\hline Hypertension (\%) & 70.9 & 32.8 & $<0.001$ \\
\hline Diabetes mellitus (\%) & 32.3 & 12.7 & $<0.001$ \\
\hline Dyslipidemia (\%) & 37.5 & 27.6 & $<0.001$ \\
\hline \multicolumn{4}{|l|}{ Medication } \\
\hline Hypertension (\%) & 58.0 & 23.5 & $<0.001$ \\
\hline Diabetes mellitus (\%) & 18.9 & 7.5 & $<0.001$ \\
\hline Dyslipidemia (\%) & 36.0 & 22.8 & $<0.001$ \\
\hline Smoking status & & & 0.119 \\
\hline Current smoker (\%) & 12.7 & 11.6 & \\
\hline Ex-smoker (\%) & 43.2 & 39.9 & \\
\hline Never smoker (\%) & 44.1 & 48.5 & \\
\hline Current drinker (\%) & 74.3 & 73.0 & 0.673 \\
\hline Regular exercise (\%) & 34.5 & 35.5 & 0.654 \\
\hline
\end{tabular}

Data are shown as the mean and (SD) or percentages

MS metabolic syndrome, SBP systolic blood pressure, DBP diastolic blood pressure, HbA1c glycated hemoglobin, HOMA-IR homeostasis model assessment of insulin resistance, HOMA- $\beta$ homeostasis model assessment of pancreatic $\beta$-cell function, $\gamma G T \gamma$-glutamyl transpeptidase, $H D L$ high-density lipoprotein, $L D L$ low-density lipoprotein, $h s C R P$ high sensitivity C-reactive protein

a Log-transformed values were used for analysis

Their mean age was about 56 years and similar between the MS group and those without MS. The subjects with MS had higher BMI, larger waist circumference, and higher blood pressure, as expected with the definition of MS.

The MS group had a significantly greater impairment in glucose tolerance and higher insulin resistance than did the group without MS $(P<0.05)$. The HOMA- $\beta$ results were not different between the two groups. Liver enzyme activities such as the levels of AST, ALT, and $\gamma$ GT were significantly higher in the MS group than in the group without it $(P<0.05)$. Serum triglyceride levels were higher and HDL-cholesterol levels were significantly lower in the MS group than in the groups without MS (both $P<0.05$ ). Serum LDL-cholesterol levels were significantly higher in the group without MS, but there lower LDL-cholesterol level in the MS group could be attributed to their used of medication. The rates of hypertension, DM, and dyslipidemia were significantly higher in the MS group than in the group without MS. The proportions of subjects who were taking medications for hypertension, DM, and dyslipidemia were also significantly greater in the MS group than in the group without MS. Smoking status, alcohol consumption, and exercise habits did not differ between the two groups.

\section{Cardiac CT findings according to the presence of metabolic syndrome}

At baseline, the mean \pm SD of CACS in the MS group was significantly greater than that in the group without MS $(103.7 \pm 270.9$ vs. $71.3 \pm 225.1, P<0.01)$. The proportion of subjects with coronary calcium $($ CACS $>0$ ) was also higher in the MS group than in the group without MS (54.4 vs. $45.8 \% ; P<0.001)$.

In the MS group, $52.6 \%$ of subjects showed CACS progression, while in the group without MS, $40.4 \%$ of subjects had progressed $(P<0.001)$. In the subgroup of 1079 subjects with data concerning the degree of coronary artery stenosis and coronary plaque using CCTA at baseline, significantly more subjects with MS had significant stenosis (13.6 vs. $8.9 \% ; P=0.017)$, multivessel involvement ( 4.2 vs. $1.7 \% ; P=0.015$ ), and coronary plaques (55.1 vs. $43.3 \%$; $P<0.001$ ) than did those without MS. In the follow-up, the subjects with MS showed a trend of progression of stenosis than did the group without MS (28.6 vs. $23.4 \% ; P=0.067$ ). Furthermore, the development of vulnerable plaque (mixed or noncalcified types) was larger in the MS group than group without MS with borderline significance (Table 2).

Next, we evaluated any association between the number of MS components and progression of CAD. As the number of MS components increased, so did the progression of CACS. The proportion of subjects with CACS progression increased gradually from $30.9 \%$ in subjects without any MS component to $66.0 \%$ in those with all five components ( $P$ for trend $<0.001$; Fig. $2 \mathrm{a}$ ). A similar 
Table 2 Comparison of cardiac computed tomography findings between subjects with and without metabolic syndrome (MS)

\begin{tabular}{|c|c|c|c|}
\hline & MS & Non-MS & $P$ \\
\hline \multicolumn{4}{|l|}{ Baseline data } \\
\hline CACS & $n=825$ & $n=1601$ & \\
\hline Initial CACS, median (IQR) & $11.1(0-98.5)$ & $0.0(0-43.0)$ & $<0.001^{\mathrm{a}}$ \\
\hline Prevalence of coronary calcification, n (\%) & $449(54.4)$ & $734(45.8)$ & $<0.001$ \\
\hline CCTA & $n=381$ & $n=698$ & \\
\hline Significant stenosis, n (\%) & $52(13.6)$ & $62(8.9)$ & 0.017 \\
\hline Multivessel disease, n (\%) & $16(4.2)$ & $12(1.7)$ & 0.025 \\
\hline Any plaque, n (\%) & $210(55.1)$ & $302(43.3)$ & $<0.001$ \\
\hline \multicolumn{4}{|l|}{ Plaque type } \\
\hline Calcified, n (\%) & $156(40.9)$ & $233(33.4)$ & 0.463 \\
\hline Mixed, n (\%) & $60(15.7)$ & $82(11.7)$ & 0.764 \\
\hline Non-calcified, n (\%) & $41(10.8)$ & $63(9.0)$ & 0.739 \\
\hline \multicolumn{4}{|l|}{ Follow up data } \\
\hline CACS & $n=825$ & $n=1601$ & \\
\hline FU interval, median (IQR), days & $1285(763.3-1640.3)$ & $1292(763.5-1703.0)$ & 0.808 \\
\hline Follow-up CACS, median (IQR) & $45.3(0-225.3)$ & $10.4(0-115.4)$ & $<0.001^{\mathrm{a}}$ \\
\hline Progression of CACS, n (\%) & $434(52.6)$ & $647(40.4)$ & $<0.001$ \\
\hline CCTA & $n=381$ & $n=698$ & \\
\hline FU interval, median (IQR), days & $1106(735.0-1484.6)$ & $1102(741.8-1465.3)$ & 0.948 \\
\hline Progression of stenosis, n (\%) & $109(28.6)$ & $163(23.4)$ & 0.067 \\
\hline Development of vulnerable plaque, n (\%) & $95(24.9)$ & $138(19.8)$ & 0.053 \\
\hline
\end{tabular}

CCTA coronary computed tomography angiography, CACS coronary artery calcium score, FU follow-up, IQR interquartile range

${ }^{\text {a } L o g-t r a n s f o r m e d ~ v a l u e s ~ w e r e ~ u s e d ~ f o r ~ a n a l y s i s ~}$

trend was observed when the progression was evaluated using the degree of coronary artery stenosis $(P$ for trend $=0.001$; Fig. $2 \mathrm{~b}$ ) or the development of vulnerable plaque ( $P$ for trend $=0.005$; Fig. $2 \mathrm{c}$ ).

Multivariable analysis of primary and secondary outcomes We performed multivariable analyses with Cox proportional hazard models to investigate any independent effect of MS on the progression of CAD (Table 3). First, the presence of MS was significantly associated with the progression of CACS after adjusting for age and sex (hazard ratio [HR], 1.68, $95 \%$ confidence interval [CI], 1.39-2.03, $P<0.001$ ) (Model A) and additional adjusting for smoking habit and family history of CAD (HR 1.67; 95 \% CI, 1.38-2.20: $P<0.001$ ) (Model B). After further adjusting for BMI, and the levels of LDL-cholesterol and HbA1c, the presence of MS was significantly linked with the progression of CACS with slight attenuation (HR 1.32; 95 \% CI 1.06-1.64; $P=0.012$ ) (Model C).

Next, we performed the same analysis using CCTA data. The presence of MS was significantly associated with the progression of coronary artery stenosis or the development of vulnerable plaque after adjusting for age and sex (HR 1.45; $95 \%$ CI 1.06-1.99; $P=0.021$ )
(Model A) and additional adjusting for smoking habit and a family history of CAD (HR 1.44; 95 \% CI 1.051.99; $P=0.025$ ) (Model B). After further adjusting for BMI, and the levels of LDL-cholesterol and HbA1c, a significant association of the presence of MS with the progression of coronary artery stenosis or development of vulnerable plaque was maintained (HR 1.47; $95 \% \mathrm{CI}$ 1.13-2.15; $P=0.045)$ (Model C).

\section{Discussion}

In this large retrospective longitudinal study, the subjects with MS had more CAD than those without MS at baseline. More importantly, those with MS showed rapid development or progression of CACS, coronary artery stenosis, and vulnerable plaque in the longitudinal follow-up, with a positive association with the number of MS components. MS was found to be an independent predictor for CAD progression even after adjusting for multiple relevant risk factors.

The CACS, one of the indicators of subclinical CAD, is correlated strongly with the extent of atherosclerosis [9]. According to the Jackson Heart Study, the presence of a high CACS was directly associated with the incidence of cardiovascular disease [38]. High CACS 


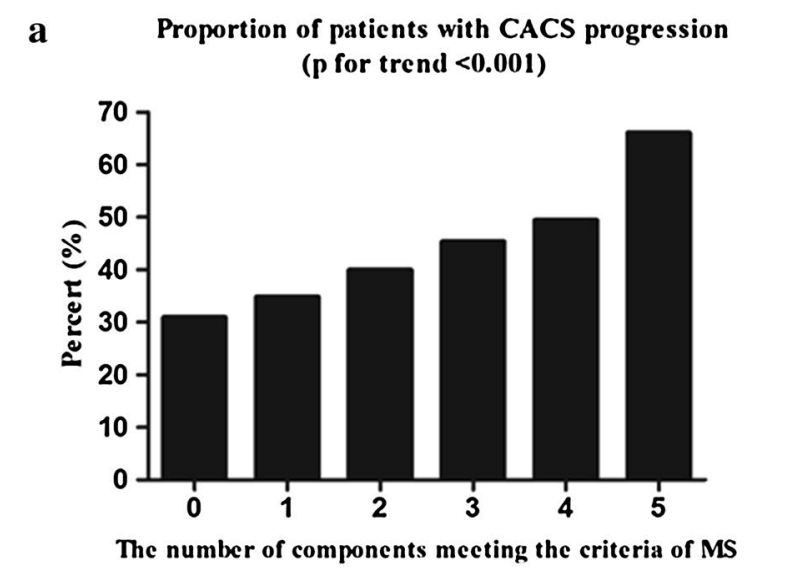

b Proportion of patients with coronary atenosis progression ( $p$ for trend $\mathbf{0 . 0 0 1}$ )

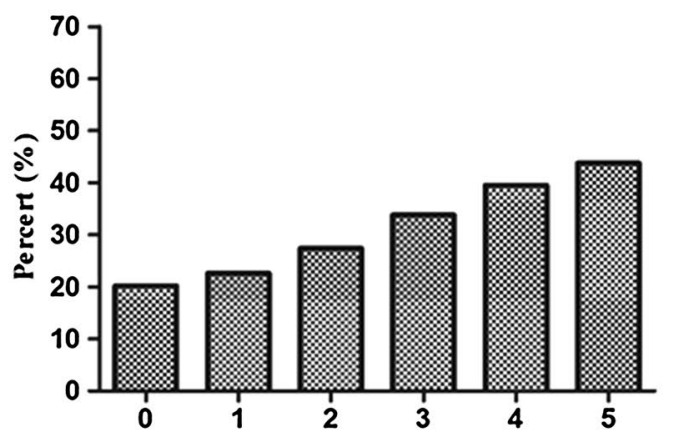

The number of components meeting the criteria of MS

c Proportion of the development of vulnerable plaue ( $p$ for trend $=0.005$ )

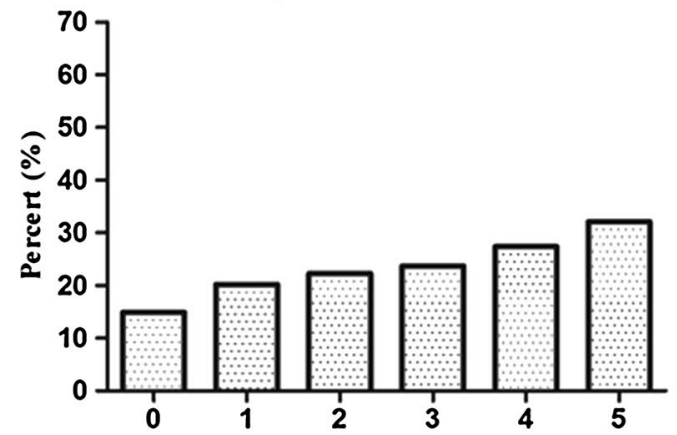

The number of components meeting the criteria of MS

Fig. 2 Proportions of CAD progression according to the number of the metabolic syndrome (MS) components present. a Proportion of patients with CACS progression. b Proportions of progression evaluated by the degree of coronary stenosis. c Proportions of the development of vulnerable plaque, according to the numbers of components of MS

was associated with arterial stiffness measured by aortic pulse wave velocity, which is another potential predictor of cardiovascular events [39]. There have been many cross-sectional studies investigating the association between MS and the high burden of coronary artery calcium [40-44]. Wong et al. [45] showed that individuals with MS had a greater degree of progression of CACS compared with those without MS. Moreover, it was reported that nonalcoholic fatty liver disease, which is now recognized as the hepatic manifestation of MS and insulin resistance, has a significant association with CACS [46, 47]. These studies suggest that CACS is an effective marker for evaluating $\mathrm{CAD}$ and further forms of CVD, as mentioned above.

However, measurement of calcium deposition only in coronary arteries seems not sufficient to evaluate CAD precisely. Even though CACS is an established surrogate marker for coronary atherosclerosis [13], it cannot provide detailed information on CAD such as the degree of coronary artery stenosis or plaque characteristics. Direct assessment of individual coronary vessels seems to be more helpful for assessing coronary vascular health than simple calcium deposition. Detailed evaluation of plaque characteristics is also of importance in this context. In fact, mixed or noncalcified plaques occur more frequently in patients with an acute coronary syndrome than in those with stable angina [48], and are known to be associated with a higher all-cause mortality outcome than calcified plaques [49]. These findings suggest that vulnerable plaques might trigger plaque rupture and are more associated with poor cardiovascular outcome [32].

So far, only a few studies have shown an association between MS and the degree of stenosis/plaque characteristics assessed by CCTA $[20,50]$. We have reported previously that MS was significantly associated with noncalcified/mixed plaques in a large number of study participants, suggesting that CCTA is a useful tool in the detailed evaluation of subclinical coronary atherosclerosis for individuals at high risk [20]. Furthermore, few studies have evaluated the influence of MS on the development or progression of CAD using CCTA in longitudinal studies [25]. To the best of our knowledge, our study is the largest that has investigated the influence of MS on development and/or progression of CAD as assessed comprehensively by CCTA in a longitudinal setting.

Here, we confirmed previous reports by demonstrating that people with MS had a greater extent of coronary calcification and a higher prevalence of significant coronary stenosis, multivessel involvement, and coronary plaques than those without MS [40-43]. Furthermore, we found a significant rate of progression of CACS in the followup. Significantly more progression of coronary artery stenosis or greater development of vulnerable plaque was also found in the subjects with MS than those without MS in the follow-up with CCTA. Among all the subjects 
Table 3 Association of metabolic syndrome with progression of CAD after multivariable adjustment

\begin{tabular}{|c|c|c|c|c|c|c|}
\hline & \multicolumn{3}{|c|}{ Progression of CACS } & \multicolumn{3}{|c|}{$\begin{array}{l}\text { Progression of coronary artery stenosis } \\
\text { or development of vulnerable plaque }\end{array}$} \\
\hline & HR & $95 \% \mathrm{Cl}$ & $P$ & HR & $95 \% \mathrm{Cl}$ & $P$ \\
\hline \multicolumn{7}{|l|}{ Model A } \\
\hline Adjusted for age and sex & 1.68 & $1.39-2.03$ & $<0.001$ & 1.45 & $1.06-1.99$ & 0.021 \\
\hline \multicolumn{7}{|l|}{ Model B } \\
\hline Adjusted for age, sex, smoking, and family history of CAD & 1.67 & $1.38-2.20$ & $<0.001$ & 1.44 & $1.05-1.99$ & 0.025 \\
\hline \multicolumn{7}{|l|}{ Model C } \\
\hline $\begin{array}{l}\text { Adjusted for age, sex, smoking, family history } \\
\text { of CAD, BMI, LDL-cholesterol, and } \mathrm{HbA} 1 \mathrm{C}\end{array}$ & 1.32 & $1.06-1.64$ & 0.012 & 1.47 & $1.01-2.15$ & 0.045 \\
\hline
\end{tabular}

$B M I$ body mass index, $C A D$ coronary artery disease, $C A C S$ coronary artery calcium score, $H b A 1 c$ glycated hemoglobin, $L D L$ low-density lipoprotein

a Vulnerable plaques contain mixed and noncalcified plaques

( $n=2426)$ enrolled in this study, 1079 underwent CCTA tests both at baseline and at least 1 year later. Of these subjects, 155 (14.4\%) had significant progression of coronary artery stenosis and/or development of vulnerable plaques without CACS progression. This finding supports our conclusion that CCTA is able to provide more sensitive information about CAD progression than simple CACS.

This study had several strengths. First, comprehensive evaluations including medical history, anthropometric measurements, and biochemical parameters were possible because data were collected through the general health check-up. Second, we analyzed long-term followup data, so that we could determine any independent association of MS with the progression of CAD. Third, we analyzed detailed information about the degree of coronary stenosis and plaque characteristics using CCTA with advanced technology.

Our study also had several limitations. First, it was a retrospective, longitudinal study so there might have been confounders that were not corrected sufficiently. Second, the study participants, who voluntarily underwent a health check-up, might not have been representative of the general Korean population. Also, their health-seeking behavior and the interventions possibly taken after the baseline evaluation might have changed their cardiovascular risk profiles during the study period and could attenuate the relationship between MS and the progression of CAD. Third, a comprehensive evaluation of CAD using CCTA was available in only $44.5 \%$ of the study participants.

\section{Conclusions}

We confirmed that MS is associated with coronary atherosclerosis as evidenced by significant coronary artery stenosis, multivessel involvement, and/or high plaque burden as well as high CACS. Subjects with MS showed greater progression not only in the CACS, but also in the degree of coronary stenosis and vulnerable plaque formation. Thus, our study has confirmed that MS is an independent risk factor for the progression of CAD even after adjusting for known cardiovascular risk factors. These findings suggest that more vigilant screening for subclinical CAD is helpful in individual with MS and more aggressive management for cardiovascular risk factors is required to prevent any progression of CAD in these subjects.

\section{Additional file}

Additional file 1: Table S1. Comparison of baseline clinical and biochemical characteristics by genders. Figure S1. Progression of coronary artery disease from baseline cardiac computed tomography scans to 3-4 years follow-up scans. 1) Increase of coronary artery calcium deposition (A and $\mathbf{B}), 2$ ) progression of coronary artery stenosis (C and $\mathbf{D}), 3$ ) development of noncalcified plaque (E and $\mathbf{F})$ 3-4 years.

\section{Authors' contributions}

LKK and JWY take full responsibility for the data collection and integrity of the analyses. SL and S-YC contributed to the hypothesis, participated in its design. JWY and LKK drafted the manuscript. D-HL, KMK, M-KK, HEP participated in the design of the study and performed the statistical analysis. SHC, KSP, HCJ, HEP, M-KK, SL and S-YC reviewed/edited the manuscript and contributed to discussion. S-YC and SL is the guarantor for the article and all authors have read and agreed the manuscript as written. All authors read and approved the final manuscript.

\section{Author details \\ ${ }^{1}$ Internal Medicine, Seoul National University College of Medicine, Seoul National University Hospital, 101, Daehak-ro, Jongno-gu, Seoul, South Korea. ${ }^{2}$ Internal Medicine, Seoul National University Bundang Hospital, 300 Gumi-dong, Bundang-Gu, Seongnam 463-707, South Korea. ${ }^{3}$ Internal Medicine, Seoul National University Hospital Healthcare System Gangnam Center, 39th FL. Gangnam Finance Center, 737 Yeoksam-dong, Gangnam-gu, Seoul 135-984, South Korea.}

\section{Acknowledgements}

We would like to thank the participants and staff for their important contributions. The first two authors contributed equally to this work. The authors assume full responsibility for analyses and interpretation of these data. 


\section{Competing interests}

The authors declare that they have no competing interests.

\section{Funding}

This research was supported by a grant from Seoul National University Hospital 0420130960 (2013-1243) and a grant from the Seoul National University Bundang Hospital.

Received: 18 March 2016 Accepted: 26 May 2016 Published online: 28 June 2016

\section{References}

1. Mendis S, Puska P, Norrving B. Global atlas on cardiovascular disease prevention and control. Geneva: World Health Organization; 2011.

2. World Health Organization. Disease and injury country estimates. Global burden of disease. Accessed from WHO website on 12th Feb. 2012.

3. Finegold JA, Asaria P, Francis DP. Mortality from ischaemic heart disease by country, region, and age: statistics from World Health Organisation and United Nations. Int J Cardiol. 2013;168(2):934-45

4. Resnick HE, Lindsay RS, McDermott MM, Devereux RB, Jones KL, Fabsitz $R R$, et al. Relationship of high and low ankle brachial index to all-cause and cardiovascular disease mortality: the strong heart study. Circulation. 2004;109(6):733-9.

5. Heald CL, Fowkes FG, Murray GD, Price JF. Risk of mortality and cardiovascular disease associated with the ankle-brachial index: systematic review. Atherosclerosis. 2006;189(1):61-9.

6. Howard BV, Wylie-Rosett J. Sugar and cardiovascular disease: a statement for healthcare professionals from the Committee on nutrition of the council on nutrition, physical activity, and metabolism of the American Heart Association. Circulation. 2002;106(4):523-7.

7. Bots ML, Hoes AW, Koudstaal PJ, Hofman A, Grobbee DE. Common carotid intima-media thickness and risk of stroke and myocardial infarction: the Rotterdam study. Circulation. 1997:96(5):1432-7.

8. von Scholten BJ, Reinhard H, Hansen TW, Lindhardt M, Petersen CL, Wiinberg $\mathrm{N}$, et al. Additive prognostic value of plasma N-terminal pro-brain natriuretic peptide and coronary artery calcification for cardiovascular events and mortality in asymptomatic patients with type 2 diabetes. Cardiovasc Diabetol. 2015;14:59.

9. Greenland P, LaBree L, Azen SP, Doherty TM, Detrano RC. Coronary artery calcium score combined with Framingham score for risk prediction in asymptomatic individuals. JAMA. 2004;291(2):210-5.

10. Polonsky TS, McClelland RL, Jorgensen NW, Bild DE, Burke GL, Guerci AD, et al. Coronary artery calcium score and risk classification for coronary heart disease prediction. JAMA. 2010;303(16):1610-6.

11. Bonow RO. Clinical practice. Should coronary calcium screening be used in cardiovascular prevention strategies? N Engl J Med. 2009;361(10):990-7.

12. Greenland $\mathrm{P}$, Bonow RO, Brundage BH, Budoff MJ, Eisenberg MJ, Grundy SM, et al. ACCF/AHA 2007 clinical expert consensus document on coronary artery calcium scoring by computed tomography in global cardiovascular risk assessment and in evaluation of patients with chest pain: a report of the American College of Cardiology Foundation Clinical Expert Consensus Task Force (ACCF/AHA Writing Committee to Update the 2000 Expert Consensus Document on Electron Beam Computed Tomography) developed in collaboration with the Society of Atherosclerosis Imaging and Prevention and the Society of Cardiovascular Computed Tomography. J Am Coll Cardiol. 2007:49(3):378-402.

13. Greenland P, Alpert JS, Beller GA, Benjamin EJ, Budoff MJ, Fayad ZA, et al. 2010 ACCF/AHA guideline for assessment of cardiovascular risk in asymptomatic adults: a report of the American College of Cardiology Foundation/American Heart Association Task Force on practice guidelines. J Am Coll Cardiol. 2010;56(25):e50-103.

14. Budoff MJ, Hokanson JE, Nasir K, Shaw LJ, Kinney GL, Chow D, et al. Progression of coronary artery calcium predicts all-cause mortality. JACC Cardiovasc Imaging. 2010;3(12):1229-36.

15. Raggi P, Callister TQ, Shaw LJ. Progression of coronary artery calcium and risk of first myocardial infarction in patients receiving cholesterol-lowering therapy. Arterioscler Thromb Vasc Biol. 2004;24(7):1272-7.
16. McEvoy JW, Blaha MJ, Defilippis AP, Budoff MJ, Nasir K, Blumenthal RS, et al. Coronary artery calcium progression: an important clinical measurement? A review of published reports. J Am Coll Cardiol. 2010;56(20):1613-22

17. Leber AW, Knez A, Becker A, Becker C, von Ziegler F, Nikolaou K, et al. Accuracy of multidetector spiral computed tomography in identifying and differentiating the composition of coronary atherosclerotic plaques: a comparative study with intracoronary ultrasound. J Am Coll Cardiol. 2004:43(7):1241-7.

18. Achenbach S, Moselewski F, Ropers D, Ferencik M, Hoffmann U, MacNeill $B$, et al. Detection of calcified and noncalcified coronary atherosclerotic plaque by contrast-enhanced, submillimeter multidetector spiral computed tomography: a segment-based comparison with intravascular ultrasound. Circulation. 2004;109(1):14-7.

19. Mowatt G, Cummins E, Waugh N, Walker S, Cook J, Jia X, et al. Systematic review of the clinical effectiveness and cost-effectiveness of 64 -slice or higher computed tomography angiography as an alternative to invasive coronary angiography in the investigation of coronary artery disease. Health Technol Assess. 2008;12(17):143.

20. Lim S, Shin H, Lee Y, Yoon JW, Kang SM, Choi SH, et al. Effect of metabolic syndrome on coronary artery stenosis and plaque characteristics as assessed with 64-detector row cardiac CT. Radiology. 2011:261(2):437-45.

21. Wilson PW, Kannel WB, Silbershatz H, D'Agostino RB. Clustering of metabolic factors and coronary heart disease. Arch Intern Med. 1999;159(10):1104-9.

22. Isomaa B, Almgren P, Tuomi T, Forsen B, Lahti K, Nissen M, et al. Cardiovascular morbidity and mortality associated with the metabolic syndrome. Diabetes Care. 2001;24(4):683-9.

23. Wang TJ, Larson MG, Levy D, Benjamin EJ, Leip EP, Omland T, et al. Plasma natriuretic peptide levels and the risk of cardiovascular events and death. N Engl J Med. 2004;350(7):655-63.

24. Shaw LJ, Raggi P, Schisterman E, Berman DS, Callister TQ. Prognostic value of cardiac risk factors and coronary artery calcium screening for all-cause mortality. Radiology. 2003;228(3):826-33.

25. He ZX, Hedrick TD, Pratt CM, Verani MS, Aquino V, Roberts R, et al. Severity of coronary artery calcification by electron beam computed tomography predicts silent myocardial ischemia. Circulation. 2000;101(3):244-51.

26. Warnick GR, Knopp RH, Fitzpatrick V, Branson L. Estimating low-density lipoprotein cholesterol by the Friedewald equation is adequate for classifying patients on the basis of nationally recommended cutpoints. Clinl Chem. 1990;36(1):15-9.

27. Matthews DR, Hosker JP, Rudenski AS, Naylor BA, Treacher DF, Turner RC. Homeostasis model assessment: insulin resistance and beta-cell function from fasting plasma glucose and insulin concentrations in man. Diabetologia. 1985;28(7):412-9.

28. Agatston AS, Janowitz WR, Hildner FJ, Zusmer NR, Viamonte M Jr, Detrano R. Quantification of coronary artery calcium using ultrafast computed tomography. J Am Coll Cardiol. 1990;15(4):827-32.

29. Raff GL, Abidov A, Achenbach S, Berman DS, Boxt LM, Budoff MJ, et al. SCCT guidelines for the interpretation and reporting of coronary computed tomographic angiography. J Cardiovasc Comput Tomogr. 2009;3(2):122-36.

30. Arbab-Zadeh A, Hoe J. Quantification of coronary arterial stenoses by multidetector CT angiography in comparison with conventional angiography methods, caveats, and implications. JACC Cardiovasc Imaging. 2011;4(2):191-202.

31. Hausleiter J, Meyer T, Hadamitzky M, Kastrati A, Martinoff S, Schomig A. Prevalence of noncalcified coronary plaques by 64-slice computed tomography in patients with an intermediate risk for significant coronary artery disease. J Am Coll Cardiol. 2006;48(2):312-8.

32. Virmani R, Burke AP, Farb A, Kolodgie FD. Pathology of the vulnerable plaque. J Am Coll Cardiol. 2006:47(8 Suppl):C13-8.

33. Virmani R, Burke AP, Kolodgie FD, Farb A. Vulnerable plaque: the pathology of unstable coronary lesions. J Interv Cardiol. 2002;15(6):439-46.

34. Expert Panel on Detection E. Treatment of high blood cholesterol in A: executive summary of the third report of The National Cholesterol Education Program (NCEP) expert panel on detection, evaluation, and treatment of high blood cholesterol in adults (adult treatment panel III). JAMA. 2001;285(19):2486-97.

35. Grundy SM, Cleeman JI, Daniels SR, Donato KA, Eckel RH, Franklin BA, et al. Diagnosis and management of the metabolic syndrome: an American 
Heart Association/National Heart, Lung, and Blood Institute Scientific Statement. Circulation. 2005;112(17):2735-52.

36. World Health Organization. The Asia-Pacific perspective: redefining obesity and its treatment. Sydney: Health Commun; 2000.

37. Berry JD, Liu K, Folsom AR, Lewis CE, Carr JJ, Polak JF, et al. Prevalence and progression of subclinical atherosclerosis in younger adults with low short-term but high lifetime estimated risk for cardiovascular disease: the coronary artery risk development in young adults study and multi-ethnic study of atherosclerosis. Circulation. 2009;119(3):382-9.

38. Xanthakis V, Sung JH, Samdarshi TE, Hill AN, Musani SK, Sims M, et al. Relations between subclinical disease markers and type 2 diabetes, metabolic syndrome, and incident cardiovascular disease: the Jackson Heart Study. Diabetes Care. 2015;38(6):1082-8.

39. Lee JY, Ryu S, Lee SH, Kim BJ, Kim BS, Kang JH, et al. Association between brachial-ankle pulse wave velocity and progression of coronary artery calcium: a prospective cohort study. Cardiovasc Diabetol. 2015;14:147.

40. Seo MH, Rhee EJ, Park SE, Park CY, Oh KW, Park SW, et al. Metabolic syndrome criteria as predictors of subclinical atherosclerosis based on the coronary calcium score. Korean J Intern Med. 2015;30(1):73-81.

41. Wong ND, Sciammarella MG, Polk D, Gallagher A, Miranda-Peats L, Whitcomb B, et al. The metabolic syndrome, diabetes, and subclinical atherosclerosis assessed by coronary calcium. J Am Coll Cardiol. 2003;41(9):1547-53.

42. Ibebuogu UN, Ahmadi N, Hajsadeghi F, Ramirez J, Flores F, Young E, et al. Measures of coronary artery calcification and association with the metabolic syndrome and diabetes. J Cardiometab Syndr. 2009;4(1):6-11.

43. Chen K, Lindsey JB, Khera A, De Lemos JA, Ayers CR, Goyal A, et al. Independent associations between metabolic syndrome, diabetes mellitus and atherosclerosis: observations from the Dallas Heart Study. Diab Vasc Dis Res. 2008;5(2):96-101.
44. Taylor AJ, Wu H, Bindeman J, Bauer K, Byrd C, O'Malley PG, et al. The relationship between the cardiometabolic syndrome and coronary artery calcium progression. J Clin Hypertens (Greenwich). 2009;1 1(9):505-11.

45. Wong ND, Nelson JC, Granston T, Bertoni AG, Blumenthal RS, Carr JJ, et al. Metabolic syndrome, diabetes, and incidence and progression of coronary calcium: the multiethnic study of atherosclerosis study. JACC Cardiovasc imaging. 2012;5(4):358-66.

46. Lee MK, Park HJ, Jeon WS, Park SE, Park CY, Lee WY, et al. Higher association of coronary artery calcification with non-alcoholic fatty liver disease than with abdominal obesity in middle-aged Korean men: the Kangbuk Samsung health study. Cardiovasc Diabetol. 2015:14:88.

47. AlRifai M, Silverman MG, Nasir K, Budoff MJ, Blankstein R, Szklo M, et al. The association of nonalcoholic fatty liver disease, obesity, and metabolic syndrome, with systemic inflammation and subclinical atherosclerosis: the multi-ethnic study of atherosclerosis (MESA). Atherosclerosis. 2015;239(2):629-33.

48. Motoyama S, Kondo T, Sarai M, Sugiura A, Harigaya H, Sato T, et al. Multislice computed tomographic characteristics of coronary lesions in acute coronary syndromes. J Am Coll Cardiol. 2007;50(4):319-26.

49. Ahmadi N, Nabavi V, Hajsadeghi F, Flores F, French WJ, Mao SS, et al. Mortality incidence of patients with non-obstructive coronary artery disease diagnosed by computed tomography angiography. Am J Cardiol. 2011;107(1):10-6.

50. Ryu J, Yong HS, Huh S, Kang EY, Woo OH. Relation of coronary atherosclerosis and metabolic syndrome in asymptomatic subjects: evaluation with coronary CT angiography. Int J Cardiovasc Imaging. 2013;29(Suppl 2):101-7.

\section{Submit your next manuscript to BioMed Central and we will help you at every step:}

- We accept pre-submission inquiries

- Our selector tool helps you to find the most relevant journal

- We provide round the clock customer support

- Convenient online submission

- Thorough peer review

- Inclusion in PubMed and all major indexing services

- Maximum visibility for your research

Submit your manuscript at www.biomedcentral.com/submit
O Biomed Central 